\title{
hTERT Protein Expression in Cytoplasm and Nucleus and its Association With HPV Infection in Patients With Cervical Cancer
}

\author{
PABLO MORENO-ACOSTA ${ }^{1,2}$, MÓNICA MOLANO $^{3}$, NICOLAS MORALES ${ }^{2}$, JINNETH ACOSTA ${ }^{4}$, \\ CRISTIAN GONZÁLEZ-PRIETO ${ }^{5}$, DIANA MAYORGA ${ }^{1}$, LINA BUITRAGO $^{6}$, OSCAR GAMBOA $^{6}$, \\ JUAN CARLOS MEJÍA ${ }^{7}$, JULY CASTRO ${ }^{7}$, ALFREDO ROMERO-ROJAS ${ }^{7}$, SOPHIE ESPENEL $^{8}$, \\ GERALD L. MURRAY ${ }^{3,9,10}$, SUZANNE M. GARLAND ${ }^{3,9,10}$, ALEXIS VALLARD $^{8}$ and NICOLAS MAGNÉ ${ }^{8}$ \\ ${ }^{1}$ Research Group in Radiobiology Clinical, Molecular and Cellular, National Cancer Institute, Bogotá, Colombia; \\ ${ }^{2}$ Research Group in Cancer Biology, National Cancer Institute, Bogotá, Colombia; \\ ${ }^{3}$ Centre Women's Infectious Diseases Research, The Royal Women's Hospital, Melbourne, Australia; \\ ${ }^{4}$ Pathology Group, National University of Colombia, Bogotá, Colombia; \\ ${ }^{5}$ Statistics Department, National University of Colombia, Bogotá, Colombia; \\ ${ }^{6}$ Unit of Analysis, National Cancer Institute, Bogotá, Colombia; \\ ${ }^{7}$ Group of Pathology Oncology, National Cancer Institute, Bogotá, Colombia; \\ ${ }^{8}$ Department of Radiation Oncology, Institut de Cancérologie de la \\ Loire-Lucien Neuwirth, Saint-Priest en Jarez, France; \\ ${ }^{9}$ Department of Obstetrics and Gynecology, University of Melbourne, Parkville, VIC, Australia; \\ ${ }^{10}$ Murdoch Children's Research Institute, Parkville, VIC, Australia
}

\begin{abstract}
Background: Few studies have analyzed the association between human telomerase reverse transcriptase (hTERT) protein expression (nuclear and cytoplasmic localization), hTERT methylation status, and human papillomavirus (HPV) genotype infection in cervical cancer. Patients and Methods: One hundred seventy-three patients with cervical cancer were analyzed. hTERT protein expression was detected by immunohistochemistry. hTERT DNA methylation analysis was performed using a PCR-RLBhTERT assay, targeting two regions of the hTERT promoter. Type specific HPV infection was detected by using GP5+/GP6+PCR-RLB. Results: hTERT protein expression was found in both cytoplasm and nucleus $(78.0 \%$ of the samples showed a cytoplasmic localization and $79.8 \%$ had
\end{abstract}

This article is freely accessible online.

Correspondence to: Dr. Pablo Moreno-Acosta, Ph.D, Research Group in Radiobiology Clinical, Molecular and Cellular \& Research Group in Cancer Biology, Research Area, National Cancer Institute, Calle $1^{\text {a }}$ No 9-85, Bogotá, Colombia. Tel: +57 14320160 Ext. 4206, Fax: +57 14320160, e-mail: pmoreno@cancer.gov.co

Key Words: Cervical cancer, hTERT protein expression, hTERT localization, nuclear hTERT, cytoplasmic hTERT, hTERT methylation, HPV. a nuclear localization). A statistically significant association was found between alpha 9 and 7 HPV species with a nonmethylation pattern of the hTERT promoter and between these species and high expression of hTERT protein with nuclear localization. Conclusion: hTERT protein is found in both the nucleus and cytoplasm of patients with cervical cancer and confirm the relationship between the nonmethylated status of hTERT promoter and some HPV species as well as the relationship between these species and hTERT protein expression.

Epidemiological studies have demonstrated a strong causal relationship between persistent infection with human papillomavirus (HPV), especially high-risk HPV (HRHPV) types and the development of cervical cancer (1). In vitro studies have shown that neoplastic transformation of cells infected with HRHPV is mainly due to telomerase activation by the action of HPV E6 oncoprotein (2). Telomerase is a ribonucleoprotein complex with terminal transferase activity and is made up by an RNA component (hTR), a catalytic protein subunit (hTERT) and the telomerase associated protein TEP1 (3). Some studies have shown a significant telomerase expression and telomerase activity levels in different types of cancer (e.g. lung, pancreas, hepatocellular, prostate, skin and certain gastrointestinal tumors) and malignant cell lines, while their levels are very low in 
healthy tissues; hence it has been proposed as a tumor marker (4). Because telomerase plays a fundamental role in the processes of cellular immortalization, it has been of great importance to establish the control mechanisms on each of its components and their impact on expression levels (5-8).

Genomic instability and cancer development arises as an effect of the response to DNA damage (9). The role of telomerase in the response to DNA damage in normal human tissue is evidenced as a protective function at the ends of the telomere (10), a role that is also indirectly demonstrated when the cellular response to disruption of the doublestranded DNA is canceled due to the suppression of hTERT (11). In the context of resistance of tumor cells against DNA damage, telomerase has been reported to be cross-linked with different signaling pathways that regulate cell proliferation, repair of DNA damage and cell death, and has been shown the antiapoptotic role of hTERT (10). Thus, in the early stages of recognizing DNA damage, hTERT may contribute to the cellular response to genotoxic effects within the cell survival mechanism $(10,11)$.

Telomerase activity as expression of hTERT mRNA increases significantly according to the progression of pre malignant cervical lesions, presenting as an early event in the course of cervical cancer (12). Few reports on detection and location of the hTERT functional protein suggest that it is expressed both in the nucleus and in the cytoplasm of cancer cells and that its expression does not strictly reflect telomerase activity $(3,13,14)$. It has been shown that in benign lesions hTERT is located in the nucleus and gradually transfers to the cytoplasm in the process of malignant transformation; however, this information is still limited and therefore, it is important to determine its biological importance at the cytoplasmic level $(3,15)$.

DNA methylation is an epigenetic phenomenon modulating gene expression without affecting the DNA sequence $(16,17)$. It has been observed that DNA methylation cancer biomarkers can be used for early detection or treatment in cancer (18). In the case of hTERT, there is a divergence among studies, with some reporting a positive correlation between promoter hypermethylation, hTERT mRNA expression and telomerase activity in primary tumors from specific organs and tumor cell lines, while others have reported a partial or no correlation (19). Some studies have also shown that DNA methylation plays a role in deregulating the hTERT expression, and it is involved in the pathogenesis mediated by HRHPV in cervical cancer (13).

A previous study conducted by our research team on advanced cervical cancer showed that differences in hTERT methylation patterns in clinical samples seem to be associated with the genotype of $\operatorname{HPV}(8,17)$. However, in that study the number of samples analyzed was low and the association between hTERT methylation, hTERT protein expression (nuclear and/or cytoplasmic localization) together with its association with type specific HPV infection were not studied. The aim of this study was to perform the association between these variables in 173 patients with invasive cervical cancer. Finding a significant association between these variables in patients with advanced cervical cancer can be of great importance, both biologically and epidemiologically.

\section{Patients and Methods}

Study design, selection and patient characteristics. A cross-sectional study was performed, through which hTERT protein expression, hTERT gene methylation status, and type of HPV infection and their respective association were analyzed. 205 samples were obtained from the Functional Unit of the Gynecology and Pathology Group of the National Cancer Institute, Bogotá, Colombia [paraffin embedded tissues and fresh frozen from patients with locally advanced cervical cancer (IIB to IIIB FIGO stages)]. Quality of paraffin embedded tissue samples and frozen fresh tissue samples was evaluated by PCR for a 209 bp fragment of the $\beta$-globin gene as previously described $(8,20)$.

Analysis of the methylation status of the hTERT gene. Of the 173 cases included in this study, 105 samples had the minimal amount of DNA needed to perform methylation analysis.

Bisulfite modification. Sodium bisulfite modification, which induces chemical conversion of unmethylated cytosines into uracils, whereas methylated cytosines are protected from this conversion, was performed using the EZ DNA Methylation Kit, according to the manufacturer's guidelines (Zymo Research, Orange, CA, USA).

PCR amplification and hTERT methylation using RLB. hTERT DNA methylation analysis was performed on bisulfite modified DNA using a new PCR-RLB-hTERT methylation assay targeting two regions flanking the hTERT core promoter [region 1 (nt -208 to -1) and region $2(\mathrm{nt}+1$ to +104$)$ ] relative to first ATG $(15)$. In brief, the primers hTERT-F: 5'GTTTTGTTTTTTTATT TTTTAGTTT 3' and hTERT-R: biot 5'CCAACCCTAAAACCCAAA 3' were used to amplify a fragment of $312 \mathrm{bp}$. Thermocycler conditions were as follows: denaturation for $4 \mathrm{~min}$ at $95^{\circ} \mathrm{C}$, followed by 40 cycles of amplification consisting of $60 \mathrm{sec}$ at $95^{\circ} \mathrm{C}, 60 \mathrm{sec}$ at $53^{\circ} \mathrm{C}$, and $60 \mathrm{sec}$ at $72^{\circ} \mathrm{C}$, with a final extension of $4 \mathrm{~min}$ at $72^{\circ} \mathrm{C}(17)$. The amplification conditions resulted in biotinylated hTERT-PCR products. To test for potential amplification bias, PCRs were performed on serial dilutions of methylated DNA in unmethylated DNA $(100 \%, 75 \%, 50 \%, 25 \%, 10 \%$ and $0 \%)$. Negative controls, with all of the reaction components but devoid of DNA, and human methylated and non-methylated DNA controls (Zymo Research), were included in each experiment.

RLB analyses were performed as described elsewhere by Molano et al. (17). In brief, hTERT specific oligonucleotide probes containing a 5-amino group were covalently attached to a Biodyne $\mathrm{C}$ membrane (Pall BioSupport), in parallel lines, using a miniblotter. Four different pairs of oligonucleotide probes directed against the hTERT gene were used to identify 8 different $\mathrm{CpG}$ sites. After binding of the oligonucleotide probes, the membrane was removed from the miniblotter and rotated. The slots of the miniblotter that were perpendicular to the oligonucleotide-probe lines were filled 
with $10 \mathrm{~mL}$ of each biotinylated hTERT-PCR product generated. Hybridization was performed in the miniblotter at $50^{\circ} \mathrm{C}$ with subsequent washing temperatures at $57^{\circ} \mathrm{C}$ and the reaction was visualized by use of peroxidase- labeled streptavidin, which interacted with the biotin of the probe, followed by enhanced chemiluminescense detection, as described in detail elsewhere (17).

Immunohistochemistry for detecting hTERT expression. Immunohistochemical staining (IHQ) was performed on fresh tissue samples and tissue samples embedded in paraffin. The IHC process was carried out by using the Dako EnVision kit and Dual Link system-HRP (Agilent Technologies, Santa Clara, CA, USA). Sections of $3 \mu \mathrm{m}$ tissue were used, which were deparaffinized, rehydrated and washed. Antigenic recovery was performed by using an antigen retrieval solution $\mathrm{pH} 9,10 \mathrm{X}$ (Agilent Technologies). Tissues were placed in a $6 \% \mathrm{H}_{2} \mathrm{O}_{2}$ solution for 7 minutes to block the endogenous peroxidase and then were washed with PBS. An incubation with rabbit monoclonal antibody was performed for 45 minutes at room temperature: hTERT (mAB telomerase reverse transcriptase ab5181 abcam) at 1:20 dilution. The IHQ reading classification scales were based on what was previously reported (21). Two oncopathologists performed the evaluation of each marker. The staining intensity score was defined as: Extension of positivity (percentage of positive cells: positive + : $11-50 \%$; positive ++ : $51-80 \%$; positive +++ : $>80 \%$ ); A negative expression was defined by a staining $<10 \%$.

HPV detection. HPV DNA detection was carried out using a standard PCR-based GP5+/GP6+assay, which allows detection of a broad spectrum of genital HPV types, followed by a specific RLB. The test was used to identify 37 specific HPV types (14 high-risk types and 23 low-risk types) as described by Molano et al., (17).

Statistical analysis. For the statistical analysis, variables of interest were age, clinical stage, tumor size, and hTERT protein expression (cytoplasmic and nuclear localization), hTERT methylation, HPV infections and treatment response (hTERT protein expression were compared between complete responders versus non-responders). Shapiro Wilk's test was used to determine if continuous variables were normally distributed. The Mann Whitney non-parametric test for non-normal data was used. Fishers test was used when the size of the sample was small to test the association between two categorical variables and when the assumptions necessary to apply the chi-square test were not met. For univariate and multivariate analyses, a logistic regression was performed. A two-sided $p$-value of $<0.05$ was considered significant. Additionally, a multiple correspondence analysis was applied to determine the relationship between HPV species, methylation status, nuclear and cytoplasmic localization of the hTERT protein expression was carried out with data from 155 patients. The $\mathrm{R}$ statistical software was used for all statistical analyzes and FactoMineR-package was used for multiple correspondence analysis.

\section{Results}

Patient, clinical and treatment characteristics. A total of 173 patients with locally advanced cervical cancer were included, with a mean age of 48 years (range=23-75). Patients had FIGO IIB $(n=62,35.8 \%)$ and FIGO IIIB $(n=111,64.2 \%)$ tumors. Most of patients $(\mathrm{n}=139,80.3 \%)$ presented a
Table I. Patient characteristics.

\begin{tabular}{|c|c|c|c|}
\hline Characteristics & Mean (range) & Number & of patients $\%$ \\
\hline Age (years) & $48(23-75)$ & 173 & 100 \\
\hline \multicolumn{4}{|l|}{ FIGO stage } \\
\hline IIB & & 62 & 35.8 \\
\hline IIIB & & 111 & 64.2 \\
\hline \multicolumn{4}{|l|}{ Histological type } \\
\hline $\mathrm{SCC}$ & & 167 & 96.5 \\
\hline Adenocarcinoma & & 6 & 3.5 \\
\hline \multicolumn{4}{|l|}{ Histological differentiation } \\
\hline Well & & 10 & 5.6 \\
\hline Moderately & & 137 & 79.3 \\
\hline Poorly & & 26 & 15.1 \\
\hline \multicolumn{4}{|l|}{ Tumor size* } \\
\hline$<4 \mathrm{~cm}$ & & 11 & 5.4 \\
\hline$>4 \mathrm{~cm}$ & & 162 & 93.6 \\
\hline Hemoglobin hb (g/dl) & 12.3 & & \\
\hline $\mathrm{Hgb} \leq 11 \mathrm{~g} / \mathrm{d}$ & & 109 & 63.0 \\
\hline $\mathrm{Hgb}>11 \mathrm{~g} / \mathrm{d}$ & & 64 & 37.0 \\
\hline \multicolumn{4}{|l|}{ Keratinization } \\
\hline Present & & 129 & 74.5 \\
\hline Absent & & 41 & 23.5 \\
\hline \multicolumn{4}{|l|}{$\mathrm{KI}$} \\
\hline $70-80$ & & 34 & 19.7 \\
\hline $90-100$ & & 139 & 80.3 \\
\hline \multicolumn{4}{|l|}{ Treatment type } \\
\hline Radiotherapy & & 72 & 41.6 \\
\hline Radiotherapy + Chemotherapy & & 101 & 58.4 \\
\hline HPV & & 157 & 90.7 \\
\hline \multicolumn{4}{|l|}{ HPV species } \\
\hline Alpha 9 & & 112 & 71.3 \\
\hline Alpha7 & & 29 & 18.5 \\
\hline Alpha 6 & & 16 & 10.2 \\
\hline
\end{tabular}

FIGO: International Federation of Gynecology and Obstetrics; HPV: human papilloma virus; SSC: squamous cell carcinoma; KI: Karnosfki indice. *Tumor size: Tumor size before treatment.

Karnofsky performance scale index $>90 \%$. Patient selection and characteristics are reported in Table I. Of 173 patients, 72 patients underwent exclusive RT without concurrent chemotherapy and 101 underwent chemo-radiotherapy; only for 22 of these was its efficacy analyzed 3 months after the end of treatment. The evaluation of the quality of the paraffinembedded tissues and fresh frozen tissues of the 205 samples analyzed generated the following results: $173(84.4 \%)$ amplified a band of $209 \mathrm{bp}$ corresponding to the $\beta$-globin gene, of which $87 / 90(96.7 \%)$ corresponded to frozen tissue samples and 86/115 (74\%) to paraffin-embedded tissues.

Methylation status analysis. The methylation analysis was performed in $97.7 \%(85 / 87)$ of the frozen tissue, and $23.2 \%$ $(20 / 86)$ of the paraffin embedded tissue, after quality assessment. Of the 105 samples, $84(80 \%)$ showed a nonmethylated pattern and $21(20 \%)$ a pattern of partial methylation in the region 1 of the hTERT promoter. In region 


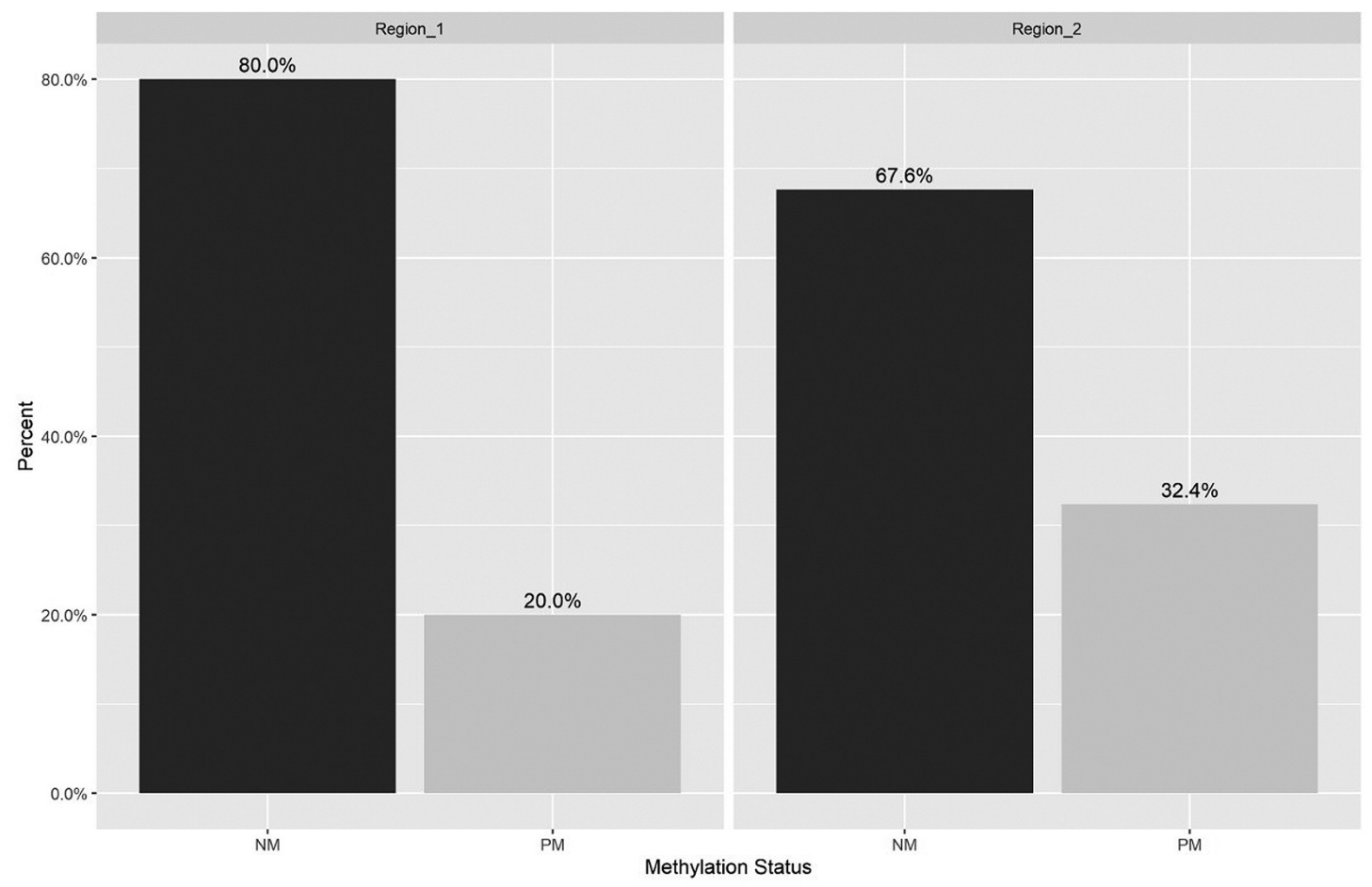

Figure 1. Methylation status of the human telomerase reverse transcriptase (hTERT) gene by region. NM: Non-methylated; PM: partial methylated; Sd: standard deviation; hTERT core promoter [Region_1 ( nt -208 to -1) NM and PM sd (0.039); Region_2 (nt +1 to + 104), NM and PM sd (0.045)]. Proportion comparison tests: Between Region_1 - NM and Region_2 - NM (p-Value=0.0596); Between Region_1 - PM and Region_2 - PM (pValue $=0.059$ ).

Table II. Human telomerase reverse transcriptase (hTERT) methylation by human papillomavirus (HPV) species.

\begin{tabular}{|c|c|c|c|c|c|}
\hline \multirow[b]{2}{*}{ Species HPV } & \multirow[b]{2}{*}{ HPV genotype } & \multicolumn{2}{|c|}{ Region 1 (nt -208 to -1 ) } & \multicolumn{2}{|c|}{ Region $2(\mathrm{nt}+1$ to +104$)$} \\
\hline & & $\mathrm{NM} \%$ & $\mathrm{PM} \%$ & NM\% & PM\% \\
\hline \multirow[t]{5}{*}{ Species alpha 9} & 16 & 77.6 & 22.4 & 65.5 & 34.5 \\
\hline & 52 & 60 & 40 & 60 & 40 \\
\hline & 35 & 75.5 & 25.5 & 75.5 & 25.5 \\
\hline & 31 & 33.3 & 66.7 & 33.3 & 66.7 \\
\hline & 58 & 100 & 0 & 100 & 0 \\
\hline \multirow[t]{3}{*}{ Species alpha 7} & 18 & 100 & 0 & 87.5 & 12.5 \\
\hline & 45 & 100 & 0 & 83.3 & 16.7 \\
\hline & 59 & 100 & 0 & 66.7 & 33.3 \\
\hline \multirow[t]{2}{*}{ Species alpha 6} & 56 & 75 & 25 & 50 & 50 \\
\hline & 66 & 100 & 0 & 0 & 100 \\
\hline
\end{tabular}

NM: No methylation; PM: partial methylation.

2, $71(67.6 \%)$ showed a non-methylated pattern and 34 $(32.4 \%)$ a partial methylation pattern (Figure 1). In the comparison tests regarding methylation status and type of region, the following results were found: For Region $1 v s$. Region 2, a trend towards statistical significance for the Non-methylation status (NM) was observed (0.059) as well as for the partially methylated (PM) status (0.059).
HPV types of alpha 7 species were associated with a lower percentage of methylation specifically in the promoter region, as compared to alpha 9 species types, where the percentages of partial methylation were higher. The highest percentage of partial methylation was observed in patients infected by HPV 31 of alpha 9 species (Table II). An association was also established between alpha 9 species and 


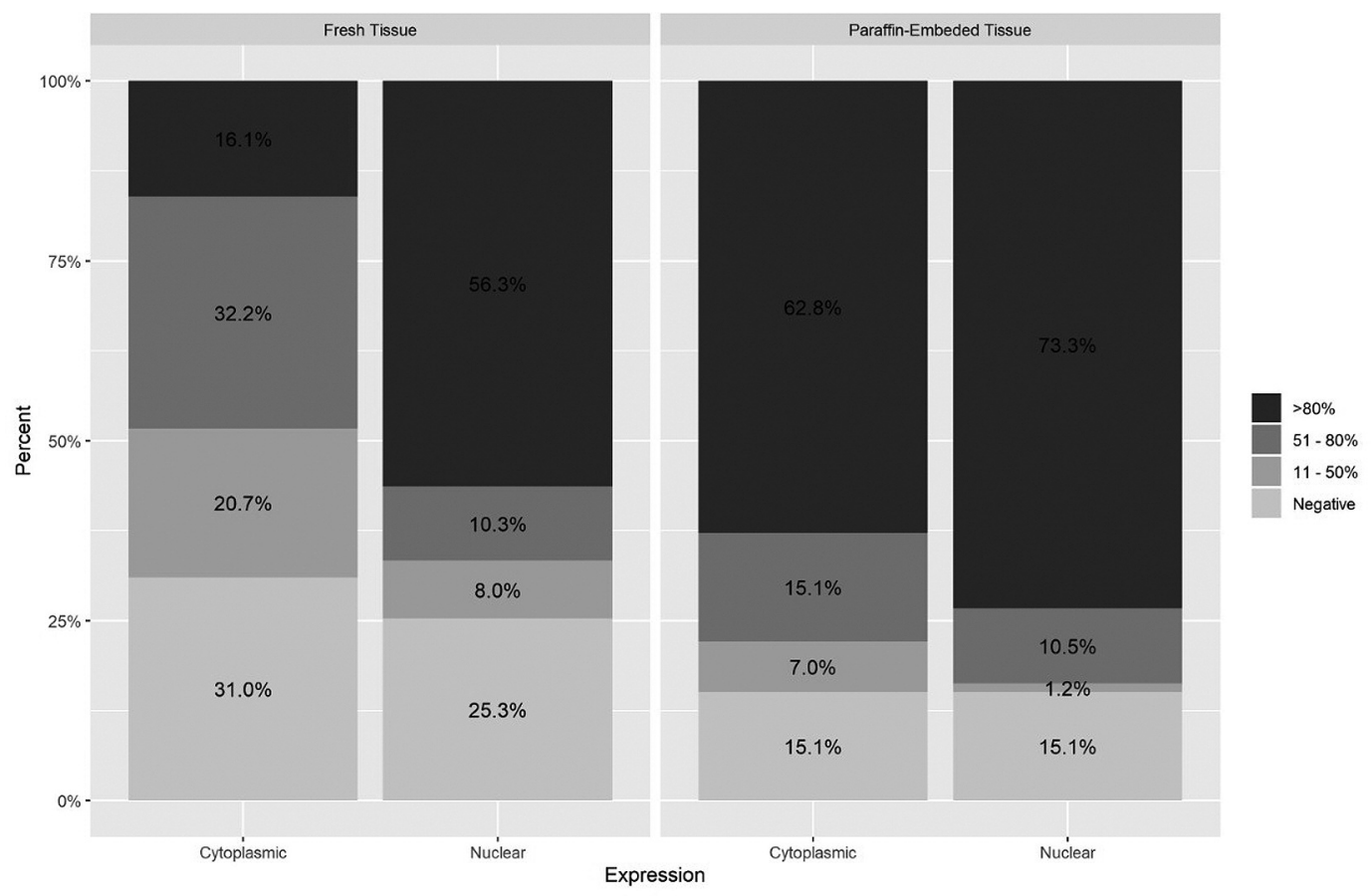

Figure 2. Human telomerase reverse transcriptase (hTERT) protein expression \& cellular location. Extension of positivity IHQ (Percentage of positive cells: positive +: 11-50\%; positive $++: 51-80 \%$; positive $+++:>80 \%)$; A negative expression was defined by a staining $<10 \%$. Ratio comparison tests-fresh tissue vs. paraffin-embedded tissue: Cytoplasmic expression $>80 \%(p=0.0001) ; 51-80 \%(p=0.0138) ; 11-50 \%(p=0.0169)$; $<10 \%$ ( $p=0.0213)$; nuclear expression $>80 \%(p=0.029) ; 51-80 \%(p=1) ; 11-50 \%(p=0.0729)$; $<10 \%(p=0.14)$.

the non-methylation status of the hTERT gene in the promoter region $(p=0.04)$, as well as with alpha 7 species $(p=0.01)$.

hTERT protein expression. Of the 173 patients that expressed hTERT protein, 135 (78.1\%) showed cytoplasmic expression and 138 showed nuclear expression (79.8\%). In fresh frozen tissue, $73 \%$ expressed hTERT $(74.7 \%$ in the nucleus and $71.3 \%$ in the cytoplasm), while $84.9 \%$ was detected in paraffin embedded tissue, both in the nucleus and cytoplasm (Figure 2). In the comparison tests regarding the expression and tissue type, the following results were found: In paraffin-embedded tissue versus fresh tissue, an association was established for cytoplasmic expression $>80 \%(p=0.0001)$ as well as for nuclear expression $>80 \%$ $(p=0.0298)$; in fresh vs paraffin tissue, an association was established for cytoplasmic expression between 51-80\% $(p=0.013), 11-50 \%(p=0.016)$ and $<10(p=0.021)$ (Figure 2 ). When performing the analysis using a positivity extension score greater than $80 \%$ and comparing nucleus and cytoplasm in both fresh and frozen tissue, we observed some differences: we identified a higher percentage of cases in nucleus than in cytoplasm, and in tissue-embedded in paraffin we saw high percentage in nucleus and cytoplasm simultaneously (Figure 3).
HPV detection. 14 HRHPV and 5 low-risk (LR) HPV types were detected and identified. Typing of 157/173 (90.7\%) samples was achieved, within which 140 (89.2\%) single infections and $17(10.8 \%)$ multiple infections were detected. In single infections, HPV type 16 was the most frequent in $82(58.6 \%)$ of the samples analyzed, followed by HPV 18 (8.6\%) and HPV58 (6.4\%). As for multiple infections, the most frequent were HPV 44, 56 and HPV 52, 66, each in $11.8 \%$ of the infections detected. HPV16 was also the most frequent type in multiple infections, followed by types 56 and 52. In relation to HPV species, alpha 9 species was the most frequent $(65.1 \%)$ followed by alpha $7(16.6 \%)$ and alpha $6(9.2 \%)$.

HPV detection and hTERT protein expression. HPV16 was detected in $84.4 \%$ and in $82.2 \%$ of cases that expressed hTERT at the nuclear level and at the cytoplasmic level respectively. The HPV species most frequently found in cases that expressed hTERT at the nuclear level and at cytoplasmic level were alpha 9 species (HPV 16, 31, 35, 52, $58 ; 80 \%$ of 102 alpha 9-positive infections), followed by alpha 7 (HPV 18, 45, 59; 83\% of 26 alpha 7-positive infections) and alpha 6 (HPVs 56, 66; 50\% of 15 alpha 6 positive infections) (Figure 3 ). The percentage of expression for hTERT in the nucleus was found to be higher in HPV 31 

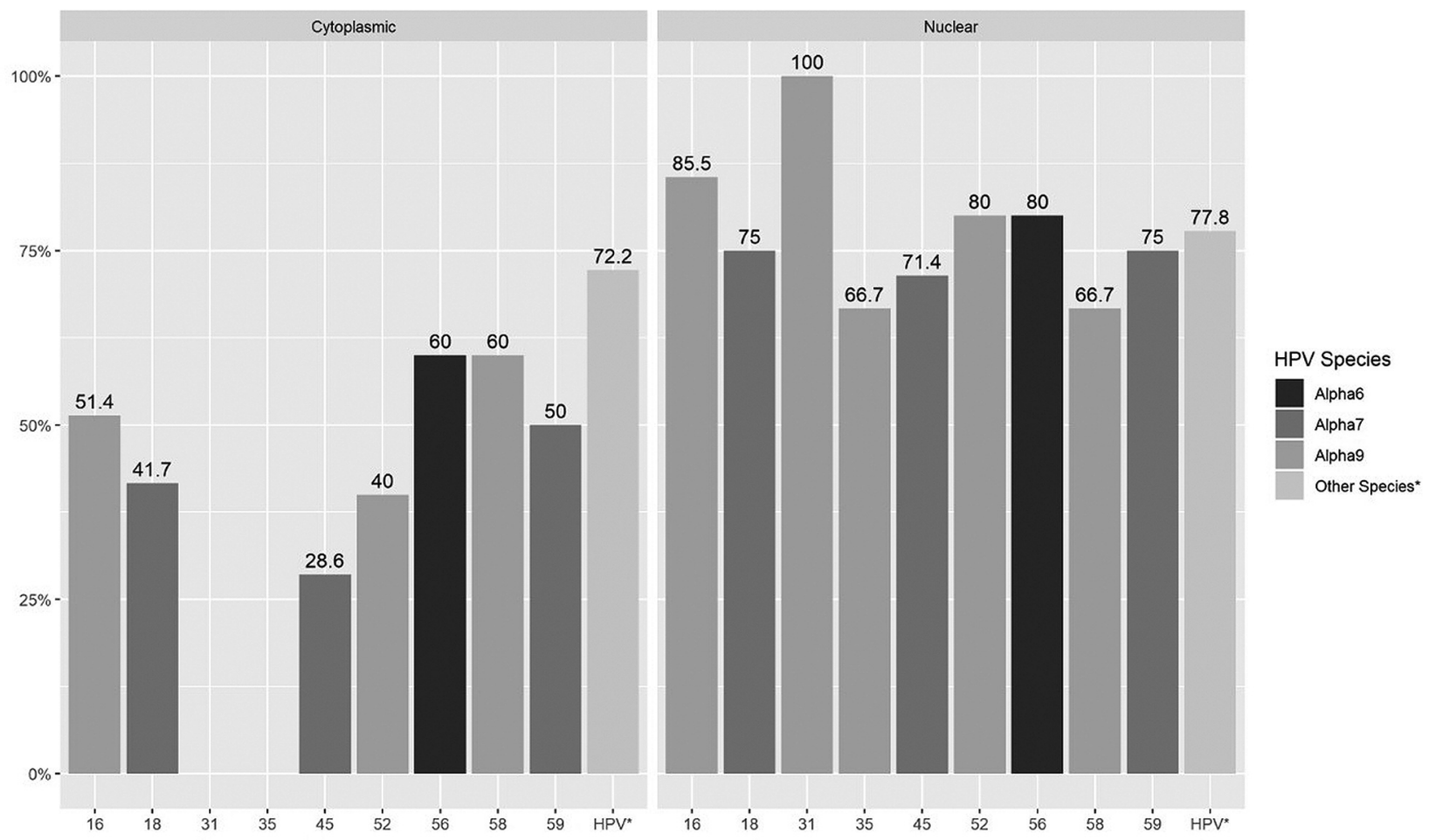

Figure 3. Human telomerase reverse transcriptase (hTERT) protein expression $>80 \%$ and human papillomavirus (HPV) species. HPV species: Alpha 9 (HPVs 16, 31, 35, 52, 58), Alpha 7 (HPVs 18, 45, 59), Alpha 6 (VPH 56). Relationship between HPV species alpha 9, alpha 7 and nuclear expression: Fisher's exact tests ( $p=0.042)$; No evidence of non-independence between the HPV species and cytoplasmic expression: Fisher's exact tests $(p=0.07)$.

cases, and in cytoplasm the percentage of expression was found to be higher in HPV 16, 56 and 58 cases, compared to the other types of HPV detected (Figure 3). A statistically significant association was found between any HPV species and hTERT expression at the nuclear level $(p=0.042)$ but not at the cytoplasm level $(p=0.07)$ (Figure 3$)$.

Our results showed a similar frequency in the level of hTERT expression in both nucleus and cytoplasm with presence of different genotypes of HPV; however, we observed that cases with levels of expression at the nucleus or cytoplasm greater than $80 \%$, showed differences in expression with respect to the type of HPV. In cases HPV31 $(+)$ and HPV35 (+) of the alpha 9 species, expression was detected only in nucleus and not in cytoplasm (Figure 3 ). In the cases with presence of other types of HPV of alpha 9, 7, 6 and others species, expression was observed both at the nucleus and cytoplasm, noting that expression levels were higher in the nucleus than in the cytoplasm (Figure 3).

Patient and clinical characteristics and hTERT expression. For the expression of the hTERT protein in cytoplasm and nucleus, no association was found with the age of the patients (Mann-Whitney test, $p=0.3532$ and $p=0.3532$ respectively), as well as for the FIGO stage (Fischer test, $p=0.684$ and $p=0.684$ respectively) and response to treatment [only 22 patients analyzed (Fischer test, $p=0.489$ and $p=0.489$ respectively)].

Multiple correspondence analysis (MCA) factor map. Correspondence analysis, which included variables such as hTERT protein expression and HPV species type, was carried out with data from 155 patients. In this analysis, MCA factor map of hTERT protein expression showing 2 dimensions explaining $75.7 \%$ of the variance. The results obtained demonstrate similarity between nuclear and cytoplasmic expression, relationship between them and the alpha 7 and alpha 9 species and likewise, a similarity between non-expression and alpha 6 species is observed (Figure 4). Besides this analysis, another MCA factor map was carried out with the data of 97 patients who, in addition to the previous variables, had data available on the methylation status for regions 1 and 2, showing 2 dimensions explaining $52.5 \%$ of the variance.; non-methylation was observed in both region 1 and region 2, but partial methylation was observed; non-methylation is more similar to alpha 7 and alpha 9 families (Figure 5). Fisher's exact tests showed a statistically significant relationship between HPV species and nuclear expression $(p=0.042)$, while with 


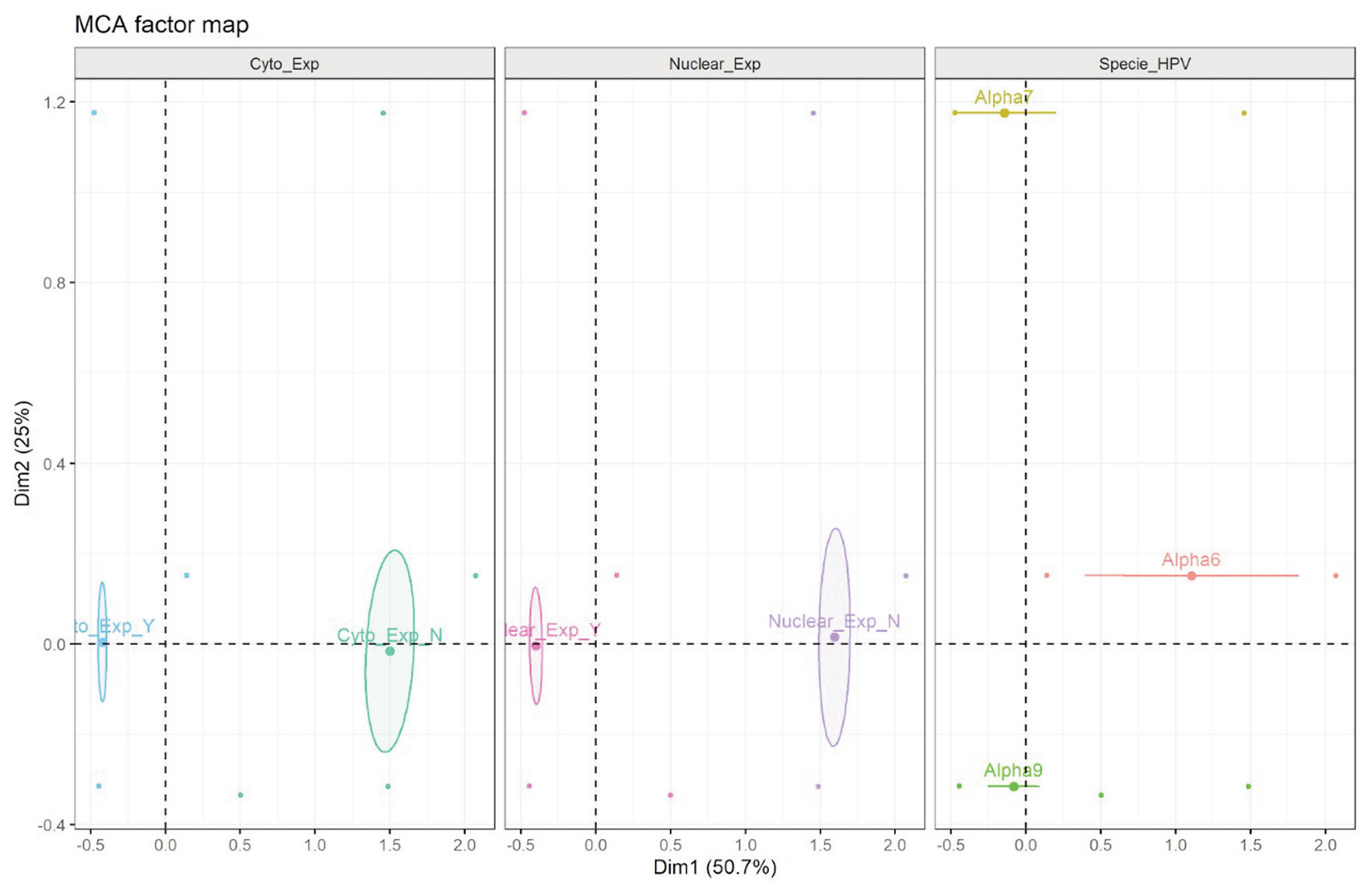

Figure 4. Multiple correspondence analysis (MCA) factor map ( $n=97$ patients included, for which all information was available). MCA of human telomerase reverse transcriptase (hTERT) protein expression showing 2 dimensions explaining $75.7 \%$ of the variance. Cyto_exp_Y $(+)$ : Cytoplasmic expression; Cyto_exp_N (+); Nuclear-exp_Y(+): Nuclear expression; Nuclear_exp_N (-). Specie_HPV: Alpha9; Alpha7; Alpha6.

$5 \%$ significance no evidence of non-independence between the HPV species and cytoplasmic expression was found $(p=0.07)$.

\section{Discussion}

This study highlights novel information regarding the association of hTERT protein subcellular localization, hTERT methylation and HPV specific infection in patients with cervical cancer. One of the most interesting finding of the present study is the subcellular localization of hTERT, which clearly demonstrated that hTERT is expressed not only at nuclear level but also at a cytoplasmic level on samples of patients with invasive cervical cancer. hTERT protein expression was detected with similar frequency in the nucleus and cytoplasm. In addition, when analysis of hTERT protein expression by using the staining intensity score with an extension of positivity $>80 \%$ expression was done, a statistically significant association between the HPV species and the expression of hTERT at the nuclear level was found $(p=0.042)$ but not a clear association at the cytoplasm level $(p=0.07)$. However, our multiple correspondence analysis also showed a close relationship between hTERT protein expression at the cytoplasm level and at the nucleus level and the alpha 9 species of HPV. Expression of the hTERT protein has been studied in chronic cervicitis, intraepithelial neoplasms and invasive cervical cancer in different studies, showing an increase in hTERT expression as the cervical disease progresses (13). This increase in hTERT expression can be due to the action of high-risk HPV E6 oncoprotein, thus mediating non-regular activities such as apoptosis blockade, gene transcription and cell proliferation. This increase could have significant functions in the viral life cycle of HPV, therefore playing an essential role in cell immortalization and in the process of malignant transformation $(2,22)$. Regarding the cellular localization of the hTERT protein, the reported findings in different tissues are few $(14,15,22-29)$. In some reports, it has been shown that although hTERT is expressed in the nucleus of benign tissues, it is gradually transferred to the cytoplasm in the process of malignant transformation $(2,22,23)$. Kio et al. were the first to report that hTERT protein detected by IHQ 


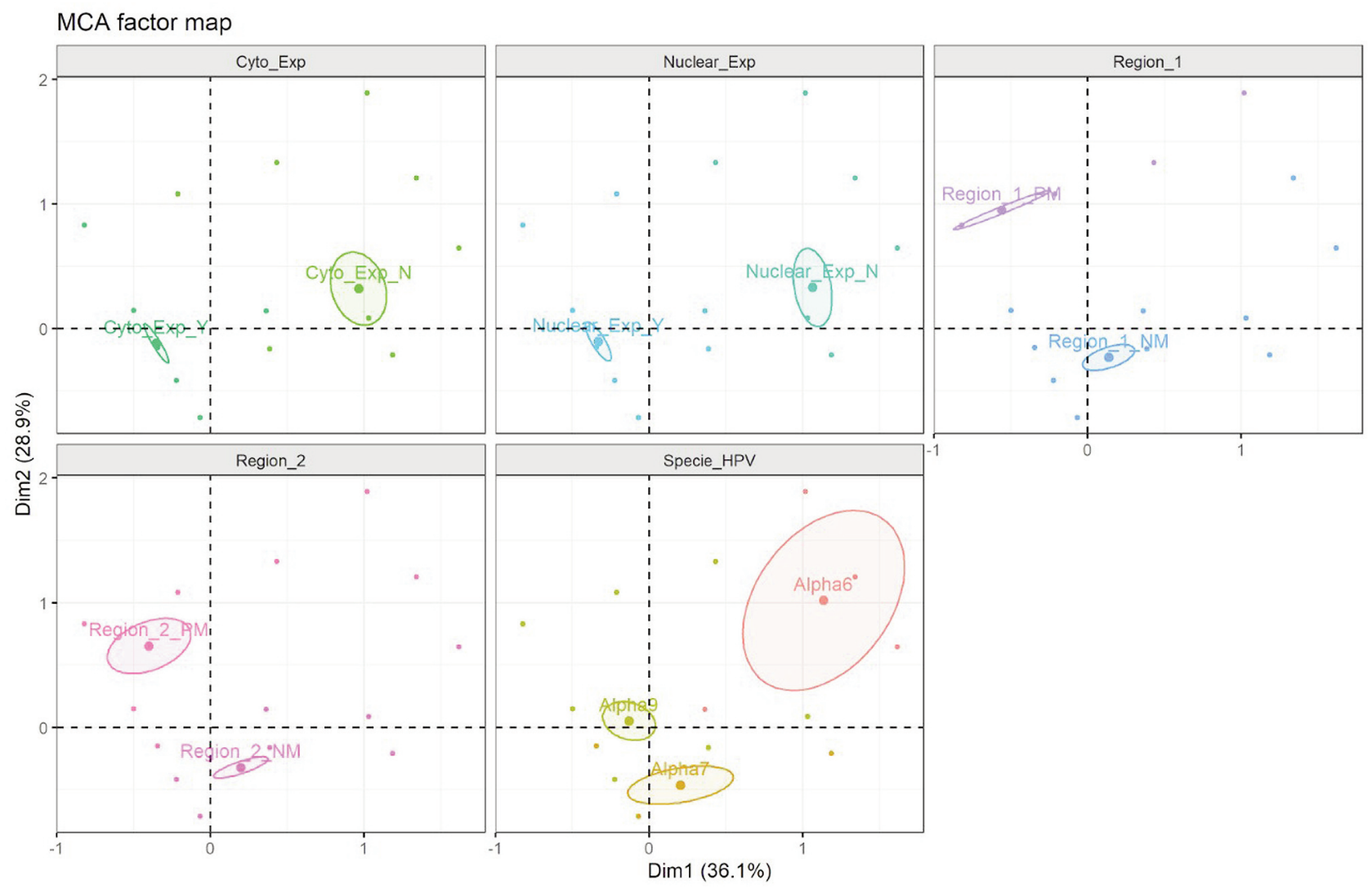

Figure 5. Multiple correspondence analysis (MCA) factor map ( $n=97$ patients included, for which all information was available). MCA of hTERT protein expression showing 2 dimensions explaining 52.5\% of the variance. Cyto_exp_Y (+); Cyto_exp_N (-); Nuclear-exp_Y (+); Nuclear_exp_N (-). Specie HPV: Alpha-9; Alpha-7; Alpha-6. Status Methylation: Region 1_NM (Not methylated); Region 1_PM (Partial methylated); Region 2_NM (Not methylated); Region 2_PM (Partial methylated).

is expressed not only in the nucleus but also in the cytoplasm of cervical cancer cells (14). This finding is similar to that reported in our present study; however, they reported a greater expression in the cytoplasm than in the nucleus, but the number of cervical cancer samples analyzed in that study was very low compared to our study (15 vs. 173 samples, respectively).

Regarding the subcellular transfer of the hTERT protein, it has been reported that it occurs from the nucleus to the mitochondria in different types of cells, including human cervical cancer, human neuroblastoma and breast adenocarcinoma cells (25). Singhapol et al. demonstrated that mitochondrial localization of TERT protects the nucleus from inflicted DNA damage and apoptosis while, in contrast, nuclear localization of TERT correlated with higher amounts of DNA damage and apoptosis (26). It has also been hypothesized that the increase in the expression of TERT in osteosarcoma cells treated with cisplatin promotes its translocation from the nucleus to the cytoplasm (27).
Based on our results and other reports, the question that has been raised about the role of the hTERT protein at the cytoplasmic level is still valid. Our results allowed us to establish a statistically significant association between the HPV species and the expression of hTERT at the nuclear level, and a trend towards statistical significance between the HPV species and the expression of hTERT at the cytoplasm level, as well as a relationship of correspondence between the expression of hTERT at level of cytoplasm and nucleus with the alpha 9 species of HPV, findings that highlight the role of HPV and hTERT in the immortalization process. Interaction between hTERT and HPV in invasive cancer could contribute to maintaining the established tumor phenotype through the deregulation or upregulation of certain genes through the avoidance of apoptosis $(2,24)$. In addition, the high levels of hTERT expression observed only at the nucleus level with the presence of high-risk HPVs such as HPV31 and HPV35 of the alpha 9 species could represent a correlation with higher amounts of DNA damage and apoptosis, strategies used by the cell to stop the cellular transformation and 
immortalization while the hTERT expression observed at the nucleus and cytoplasm in cells infected by HPV16, 18, 58 and 56 could be showing an increased immortalization process in advanced stages. High levels of hTERT expression were also observed in the nucleus rather than in the cytoplasm in the presence of HPV species Alpha 9, 7 and 6, which could be related to the upregulation of hTERT promoter activity. This type of regulation was demonstrated in vitro by the effect of HPV species such as alpha 9 and HPV 18, HPV39 and HPV51 types, according to what was reported by Van Doorslaer et al., (2). Hannen et al., demonstrated experimentally that hTERT can also modulate classic cancer pathways, such as NF signals-KB, TGF$\beta /$ Smad and Wnt, indicating that these pathways could also contribute to the metastatic potential (30).

For the analysis of the methylation status we chose two regions of the hTERT promoter due to different studies on the hTERT gene which have shown that the promoter region contains a cytosine-guanine dinucleotide (Cpg) island (CGI) and its transcriptional regulation may involve DNA methylation. A report published by our group showed that in region 1 (nt -208 to -1 ) of the central promoter $78.8 \%$ of the samples did not show methylation, while partial methylation was observed in $21.2 \%$.In region 2 (nt +1 to +104 ) of the first exon, $68.2 \%$ of the samples did not show methylation while $31.8 \%$ showed a partial methylation pattern (17). These results that were confirmed in our current study are also similar to those published Jiang et al.; they found hypomethylation of DNA around the TERT transcription start site (nt -156 to +162 ) that was functionally related to its transcription (31). Renaud et al. also proposed that hTERT promoter region is mostly non-methylated as it harbors binding sites for transcription factors that positively regulate hTERT, while the region of the first exon shows increased methylation because it is a target of a repressor protein (CTCF), indicating that this epigenetic mechanism could acts as a barrier that prevents the binding of CTCF (32). All these results support the claim that the core promoter in the region analysed (around the transcription start site) must remain nonmethylated to allow hTERT activation. However, the different correlations found between DNA methylation and TERT expression in other studies may result, in part, from the different regions analysed, variability of methods used, type of samples studied and histological diagnosis.

The analysis of methylation done in our study included both, frozen and paraffin embedded samples. When we performed the analysis of hTERT methylation in paraffin embedded samples with good quality of DNA, the results of hTERT methylation in region 1 and 2 were very similar to those obtained by using frozen samples. The use of paraffin embedded tissues and fresh frozen tissue samples together increased the power of association between HPV species and the non-methylation status. It is important to highlight that we don't recommend paraffin embedded tissues for analysis of hTERT methylation as the majority of these tissues didn't have a good enough quality of DNA compared to the high DNA quality in fresh frozen tissues. The bad quantity and quality of the DNA in the paraffin embedded tissues can be explained by the age and treatment of these tissues, added to loss of large amounts of DNA during the bisulfite conversion and cleaning procedures, so using these tissues for this type of analysis is not useful.

Regarding the relationship between the methylation status and HPV genotype infection in the immortalization stage, hTERT methylation is particularly advantageous for immortalization of cells that host oncogenic types of HPV (33). In invasive cancer, a previous report by our team showed that a non-methylation status is associated with the type of high-risk HPV present (alpha 9 and alpha 7 species) (17). Our current study strongly supported the results obtained in that report; an association between the nonmethylated status and the HPV species was also observed. These results allow us to conclude that in the presence of high-risk HPVs, the non-methylated status of hTERT predominates. These results support the claim that the core promoter must remain non-methylated to allow hTERT activation, which is consistent with the usual dogma of gene expression and epigenetic changes establishing that absence of DNA methylation around the transcription start site is necessary to allow gene expression $(8,17)$. Some studies have found that E6 proteins of different HPV types have different abilities to transactivate hTERT (2). In addition to the different E6 activation capacities of specific types and probably of the HPV species and the site-dependent methylation patterns, a wide variety of transcription factors that interact with the hTERT promoter could contribute to activating the hTERT gene (34).

A distant relationship between protein expression and methylation status was also observed in our study, as well as between non-methylation and alpha 7 and alpha 9 species of HPV. Although our numbers are few it is very interesting that samples positive for HPV 31 with only nuclear hTERT expression were the same samples that showed the higher percentage of hTERT partial methylation $(66.7 \%)$ in region 1 and region 2 of the hTERT promoter, which supports the idea that under this condition, various strategies are being used to stop or delay the cellular transformation.

In the study of correlation of clinicopathological characteristics and the result of the disease with the expression of the hTERT protein and methylation of hTERT there are some reports such as the report by Widschwendter et $a l$, in which they found that hTERT methylation in cervical cancer is significantly more frequent in comparison to normal cervical tissue, hTERT methylation increases with increasing age in ovarian cancer, hTERT expression is not correlated with prognosis whereas patients with cervical and 
ovarian cancer with non-methylated hTERT had significantly better overall survival (16). Our results regarding the clinicopathological characteristics analyzed, including response to treatment (few data were available), did not show any association with the expression of the hTERT protein, so it is necessary to carry out more studies with a larger sample size and more clinical features.

\section{Conclusion}

Our results showed the following novel and interesting findings: 1) invasive cervical cancer samples express hTERT both in the nucleus and cytoplasm; 2) hTERT protein expression by using a staining intensity score with an extension of positivity $>80 \%$ expression showed a statistically significant association between the HPV species and the expression of hTERT at the nuclear level; 3) differences in the methylation patterns of hTERT in clinical specimens of cervical cancers are associated with HPV species and specific HPV types; 4) Combination analysis of the hTERT methylation status, the hTERT subcellular protein expression and the presence of certain HPV species could be used in the future as biomarkers and molecular targets in the development of new diagnostic and treatment strategies for cervical cancer.

\section{Conflicts of Interest}

The Authors have no competing financial interests in relation to the work described.

\section{Authors' Contributions}

Conceptualization and design of the study: Molano, M., MorenoAcosta, P., Morales, N.; Methodology and statistical analysis: Moreno-Acosta, P., Molano, M., Buitrago L., González-Prieto, C., Gamboa, O., Morales, N., Mayorga, D; IHC development: Castro, J., Mejia, JC.; Reading and interpretation of molecular studies: Acosta, J., Mejia JC., Romero-Rojas A., Morales, N., MorenoAcosta, P., Molano, M.; Initial drafting of the manuscript: MorenoAcosta, P., Molano, M., Murray, G., Garland, S, Espenel, S, Vallard, A, Magné, N.; Building and final draft of the manuscript: MorenoAcosta, P., Molano, M., González-Prieto, C., Mayorga, D., Acosta, J.; Final review, addition of critical content to the discussion section and revision of all portions of the submitted manuscript: MorenoAcosta, P., Molano, M., Acosta, J., González-Prieto, C.

\section{Acknowledgements}

The Authors thank Josefa Rodriguez for the partial collection and storage of the samples.

\section{Funding}

Work funded by the National Cancer Institute (Bogota, Colombia) with the Colombian National Investment Funds.

\section{References}

1 Molano M, Martín DC, Moreno-Acosta P, Hernández G, Cornall A, Buitrago O, Gamboa O, Garland S, Tabrizi S and Muñoz N: Telomerase activity in cervical scrapes of women with high-grade cervical disease: A nested case-control study. Oncol Lett 15: 354360, 2018. PMID: 29387223. DOI: 10.3892/ol.2017.7324

2 Van Doorslaer K and Burk RD: Association between hTERT activation by HPV E6 proteins and oncogenic risk. Virology 433: 216-219, 2012. PMID: 22925336. DOI: 10.1016/j.virol.2012.08.006

3 Frost M, Bobak JB, Gianani R, Kim N, Weinrich S, Spalding DC, Cass LG, Thompson LC, Enomoto T, Uribe-Lopez D and Shroyer KR: Localization of telomerase hTERT protein and hTR in benign mucosa, dysplasia, and squamous cell carcinoma of the cervix. Am J Clin Pathol 114: 726-734, 2000. PMID: 11068546. DOI: 10.1309/XWFE-ARMN-HG2D-AJYV

$4 \mathrm{Xu} \mathrm{Y,} \mathrm{He} \mathrm{K}$ and Goldkorn A: Telomerase targeted therapy in cancer and cancer stem cells. Clin Adv Hematol Oncol 9: 442455, 2011. PMID: 21841744.

5 Taby R and Issa JP: Cancer epigenetics. CA Cancer J Clin 60: 376-392, 2010. PMID: 20959400. DOI: 10.3322/caac.20085

6 Moore LD, Le T and Fan G: DNA methylation and its basic function. Neuropsychopharmacology 38: 23-38, 2013. PMID: 22781841. DOI: $10.1038 /$ npp.2012

7 Miller J, Dakic A, Chen R, Palechor-Ceron N, Dai Y, Kallakury B, Schlegel R and Liu X: HPV16 E7 protein and hTERT proteins defective for telomere maintenance cooperate to immortalize human keratinocytes. PLoS Pathog 9(4): e1003284. 2013. PMID: 23592995. DOI: 10.1371/journal.ppat.1003284

8 Cárdenas MNM: Desarrollo de un nuevo método para el análisis del estado de metilación del gen hTERT y su asociación con la infección por el virus de papiloma humano en tejidos de pacientes con cáncer cervical invasivo. Maestría thesis, Universidad Nacional de Colombia. Available at: http://bdigital.unal.edu.co/44106/1/1019023046.2013.pdf [Last accessed on May 18 2020]

9 Gorgoulis VG, Vassiliou LV, Karakaidos P, Zacharatos P, Kotsinas A, Liloglou T, Venere M, Ditullio RA Jr, Kastrinakis NG, Levy B, Kletsas D, Yoneta A, Herlyn M, Kittas C and Halazonetis TD: Activation of the DNA damage checkpoint and genomic instability in human precancerous lesions. Nature 434: 907-913, 2005. PMID: 15829965. DOI: 10.1038/nature03485

10 Lamy E, Goetz V, Erlacher M, Herz C and Mersch-Sundermann V: hTERT: Another brick in the wall of cancer cells. Mutat Res 752: 119-128, 2013. PMID: 23287739. DOI: 10.1016/ j.mrrev.2012

11 Masutomi K, Possemato R, Wong J, Currier J, Tothova Z, Manola J, Ganesan S, Lansdorp P, Collins K and Hahn W: The telomerase reverse transcriptase regulates chromatin state and DNA damage responses. PNAS 102: 8222-8227, 2005. PMID: 15928077. DOI: $10.1073 /$ pnas.0503095102

12 Tomlinson RL, Ziegler TD, Supakorndej T and Terns RM: Cell cycle-regulated trafficking of human telomerase to telomeres. Mol Biol Cell 17: 955e965, 2006. PMID: 16339074. DOI: 10.1091/mbc.e05-09-0903

13 Cheah PL, Looi LM, Ng MH and Sivanesaratnam V: Telomerase activation and human papillomavirus infection in invasive uterine cervical carcinoma in a set of Malaysian patients. J Clin Pathol 55: 22-26, 2002. PMID: 11825919. DOI: $10.1136 /$ jcp.55.1.22 
14 Kyo S, Masutomi K, Maida Y, Kanaya T, Yatabe N, Nakamura M, Tanaka M, Takarada M, Sugawara I, Murakami S, Taira T and Inoue M: Significance of immunological detection of human telomerase reverse transcriptase: re-evaluation of expression and localization of human telomerase reverse transcriptase. Am J Pathol 163: 859-856, 2003. PMID: 12937127. DOI: $10.1016 / \mathrm{S} 0002$

15 de Wilde J, Kooter JM, Overmeer RM, Claassen-Kramer D, Meijer CJ, Snijders PJ and Steenbergen RDM: hTERT promoter activity and $\mathrm{CpG}$ methylation in HPV-induced carcinogenesis. BMC Cancer 10: 271, 2010. PMID: 20534141. DOI: 10.1186/1471-2407

16 Widschwendter A, Muller HM, Hubalek MM, Wiedemair A, Fiegl H, Goebel G, Mueller-Holznera E, Martha C and Widschwendter M: Methylation status and expression of human telomerase reverse transcriptase in ovarian and cervical cancer. Gynecol Oncol 93: 407-416, 2004. PMID: 15099954. DOI: 10.1016/j.ygyno.2004.01.036

17 Molano M, Moreno-Acosta P, Morales N, Burgos M, Buitrago L, Gamboa O, Alvarez R, Garland SM, Tabrizi SN, Steenbergen RD and Mejía JC: Association between type-specific HPV infections and hTERT DNA methylation in patients with invasive cervical cancer. Cancer Genomics Proteomics 13: 483491, 2016. PMID: 27807071. DOI: 10.21873/cgp.20011

18 Locke WJ, Guanzon D, Ma C, Liew YJ, Duesing KR, Fung KYC and Ross JP: DNA methylation cancer biomarkers: Translation to the clinic. Front Genet 10: 1150, 2019. PMID: 31803237. DOI: $10.3389 /$ fgene.2019.01150

19 Guilleret I, Yan P, Grange F, Braunschweig R, Bosman FT and Benhattar J: Hypermethylation of the human telomerase catalytic subunit (hTERT) gene correlates with telomerase activity. Int J Cancer 101: 335-341, 2002. PMID: 12209957. DOI: 10.1002/ ijc. 10593

20 Molano M, Meijer CJ, Morré SA, Pol R andvan den Brule AJ: Combination of PCR targeting the VD2 of omp1 and reverse line blot analysis for typing of urogenital Chlamydia trachomatis serovars in cervical scrape specimens. J Clin Microbiol 42: 2935-2939, 2004. PMID: 15243041. DOI: 10.1128/ JCM.42.7.2935-2939.2004

21 Moreno-Acosta P, Vallard A, Carrillo S, Gamboa O, RomeroRojas A, Molano M, Acosta J, Mayorga D, Rancoule C, Garcia MA, Cotes Mestre M and Magné N: Biomarkers of resistance to radiation therapy: a prospective study in cervical carcinoma. Radiat Oncol 12: 120, 2017. PMID: 28716107. DOI: 10.1186/s13014-017-0856-2

22 Yang H, Zhang H, Zhong Y, Wang Q, Yang L, Kang H, Gao X, Haijun Yu, Conghua X, Fuxiang $Z$ and Yunfeng Z: Concomitant underexpression of TGFBR2 and overexpression of hTERT are associated with poor prognosis in cervical cancer. Sci Rep 7: 41670, 2017. PMID: 28195144. DOI: $10.1038 /$ srep41670

23 Liu K, Hodes RJ and Weng NP: Cutting edge. Telomerase activation in human $\mathrm{T}$ lymphocytes does not require increase in telomerase reverse transcriptase (hTERT) protein but is associated with hTERT phosphorylation and nuclear translocation. J Immunol 166: 4826-4830, 2001. PMID: 28195144. DOI: 10.4049/jimmunol.166.8.4826

24 Yan P, Benhattar J, Seelentag W, Stehle JC and Bosman FT: Immunohistochemical localization of hTERT protein in human tissues. Histochem Cell Biol 121: 391-397, 2004. PMID: 15138842. DOI: $10.1007 / \mathrm{s} 00418-004-0645-5$
25 Indran IR, Hande MP and Pervaiz S: hTERT overexpression alleviates intracellular ROS production, improves mitochondrial function, and inhibits ROS-mediated apoptosis in cancer cells. Cancer Res 71(1): 266-276, 2011. PMID: 21071633. DOI: 10.1158/0008-5472.CAN-10-1588

26 Singhapol C, Pal D, Czapiewski R, Porika M, Nelson G and Saretzki GC: Mitochondrial telomerase protects cancer cells from nuclear DNA damage and apoptosis. PLoS One 8: e52989, 2013. PMID: 23326372. DOI: 10.1371/journal.pone.0052989

27 Zhang Z, Yu L, Guo Dai G, Xia K, Liu G, Qi Song Q, Tao C, Gao $\mathrm{T}$ and Guo W: Telomerase reverse transcriptase promotes chemoresistance by suppressing cisplatin-dependent apoptosis in osteosarcoma cells. Sci Rep 7(7070): 1-11, 2017. PMID: 28765565. DOI: 10.1038/s41598-017-07204-w

28 Saretzki G: Extra-telomeric functions of human telomerase: cancer, mitochondria and oxidative stress. Curr Pharm Des 40: 6386-3403, 2014. PMID: 24975608 DOI: $10.2174 / 13816128$ 20666140630095606

29 Radeghieri A, Savio G, Zendrini A, Di Noto G, Salvi A, Bergese P and Piovani G: Cultured human amniocytes express hTERT, which is distributed between nucleus and cytoplasm and is secreted in extracellular vesicles. Biochem Biophys Res Commun 483: 706711, 2017. PMID: 27988335. DOI: 10.1016/j.bbrc.2016.12.077

30 Hannen R and Bartsch JW: Essential roles of telomerase reverse transcriptase hTERT in cancer stemness and metastasis. FEBS Lett 592: 2023-2031, 2018. PMID: 29749098. DOI: $10.1002 / 1873-3468.13084$

31 Jiang J, Zhao LJ, Zhao C, Zhang G, Zhao Y, Li JR, Li XP and Wei LH: Hypomethylated $\mathrm{CpG}$ around the transcription start site enables TERT expression and HPV16 E6 regulates TERT methylation in cervical cancer cells. Gynecol Oncol 124: 534541, 2012. PMID: 22108635. DOI: 10.1016/j.ygyno.2011.11.023

32 Renaud S, Loukinov D, Abdullaev Z, Guilleret I, Bosman FT, Lobanenkov V and Benhattar J: Dual role of DNA methylation inside and outside of CTCF-binding regions in the transcriptional regulation of the telomerase hTERT gene. Nucleic Acids Res 35: 1245-1256, 2007. PMID: 17267411. DOI: 10.1093/nar/gk11125

33 Schütze DM, Kooter JM, Wilting SM, Meijer CJ, Quint W, Snijders PJ and Steenbergen RD: Longitudinal assessment of DNA methylation changes during HPVE6E7-induced immortalization of primary keratinocytes. Epigenetics 10: 73-81, 2015. PMID: 25580631. DOI: 10.4161/15592294.2014.990787

34 Veldman T, Liu X, Yuan $H$ and Schlegel R: Human papillomavirus E6 and Myc proteins associate in vivo and bind to and cooperatively activate the telomerase reverse transcriptase promoter. Proc Natl Acad Sci USA 100: 8211-8216, 2003. PMID: 12821782. DOI: 10.1073/pnas.1435900100
Received March 24, 2020

Revised May 15, 2020

Accepted May 18, 2020 